\title{
High-Speed Flame Radiation Imaging of Thermoacoustic Coupling in a High Pressure Research Thrust Chamber
}

\author{
By W. Armbruster, J. Hardi AND M. Oschwald $\dagger$ \\ Deutsches Zentrum für Luft- und Raumfahrt, Institut für Raumfahrtantriebe \\ Im Langen Grund, 74239 Hardthausen
}

This work addresses the nature of supercritical hydrogen-oxygen flame coupling to acoustics through visualization under conditions representative of upper stage rocket engines. A research thrust chamber which shows self-excited combustion instabilities for certain operating conditions was used. Previous analysis proved that the instabilities are connected to injector acoustics. In this study a small optical access window was mounted in the chamber wall. High-speed flame radiation imaging of $\mathrm{OH}^{*}$ and blue wavelengths has been applied in order to analyze the acoustic-flame interaction of one of the 42 flames. Dynamic mode decomposition results for a case with 80 bar chamber pressure showed that the flame dynamics are strongly influenced by the LOX injector acoustics, whereas for a 50 bar chamber pressure load point no flame response to injector acoustics was observed. A potential source of acoustic excitation for the oxygen injectors based on hydrodynamic effects is consistent with the contradictory behavior of the two load points.

\section{Introduction}

High-frequency combustion instabilities present a high risk for the development of liquid propellant rocket engines $[1,2]$. In general, combustion instabilities emerge if unsteady heat release is in phase with the pressure oscillation. This energy transfer is known as the Rayleigh criterion [3]. For liquid-propellant rocket engines (LPREs) the coupling mechanisms are often divided into intrinsic and injection-coupled mechanisms, in which intrinsic mechanisms are defined by processes only happening inside the combustion chamber as atomization, mixing and combustion. For injection-coupled mechanisms also the mass flow oscillations or acoustic resonance modes of the injector elements interact with the chamber acoustics [4]. For the cryogenic propellants liquid oxygen-hydrogen (LOX/H2) several examples of high-frequency combustion instability have been reported [4-7]. However, both intrinsic [5] and injection-coupled [4] mechanisms were forwarded to explain the observed instabilities.

The DLR research thrust chamber BKD allows the coupling mechanisms to be investigated under representative conditions. In previous test campaigns two different types of self-excited high-frequency combustion instability have been detected, both of which are driven by injection-coupling $[8,9]$.

The first type of instability is characterized by an excited first tangential (1T) chamber resonance mode around $10 \mathrm{kHz}$ with increased acoustic pressure amplitudes for the

$\dagger$ also at Institute of Jet Propulsion and Turbomachinery, RWTH Aachen University 
operating condition of $p_{c c}=80$ bar, $R O F=6$ and hydrogen injection temperatures of $T_{\mathrm{H} 2}=95 \mathrm{~K}$ [8]. Analysis of flame radiation in BKD with fiber optical probes helped to gain a basic understanding of the coupling mechanism of the observed combustion instabilities. The signals of the optical probes revealed that dominant frequencies which do not correspond to chamber acoustic modes are present in the $\mathrm{OH}^{*}$ signals. This type of instability is presented in Fig. 1 in which spectrograms of dynamic chamber pressure oscillation and $\mathrm{OH}^{*}$ fluctuation are compared for a typical test sequence of previous BKD test campaigns. The dominant $\mathrm{OH}^{*}$ frequencies were later identified to be generated by
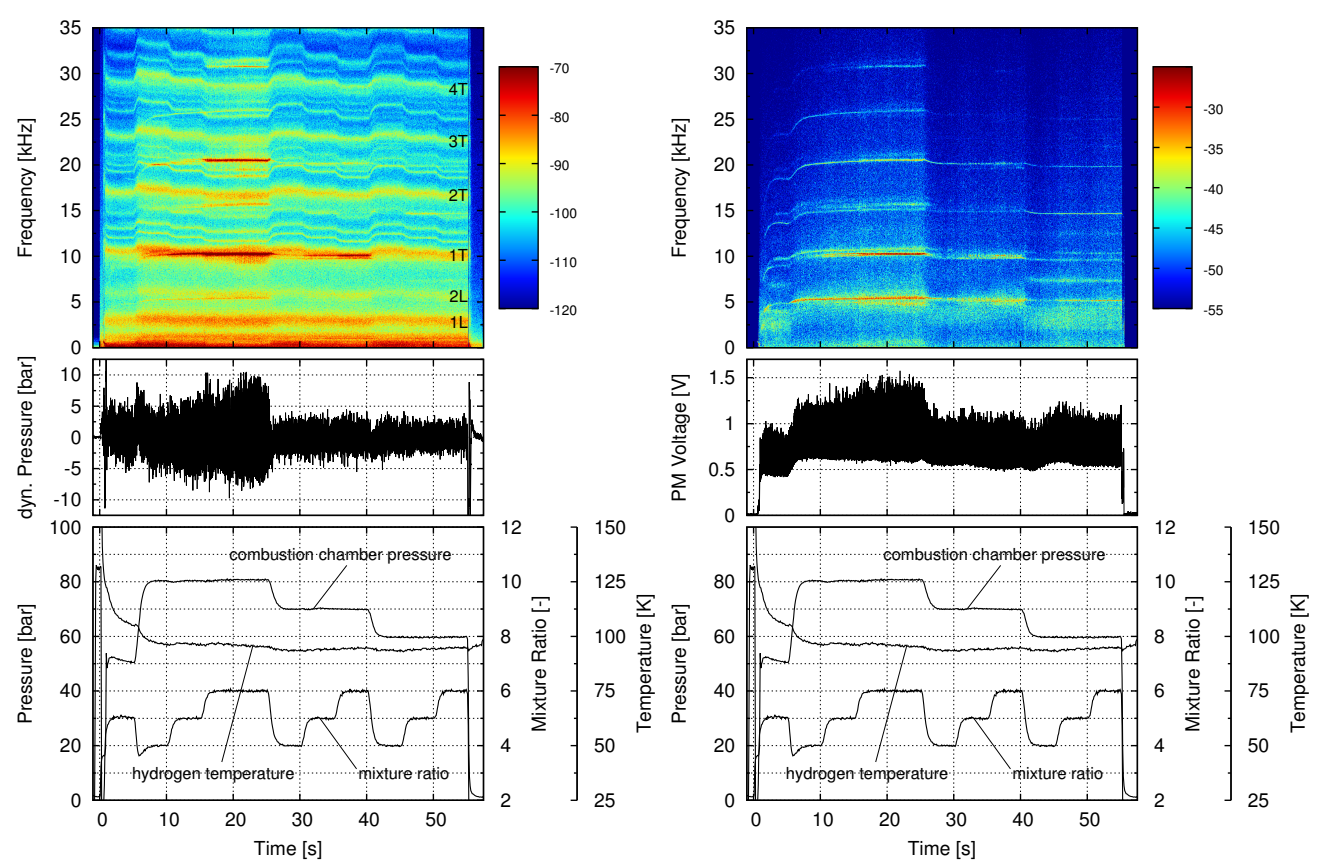

FIGURE 1. Spectrogram and raw signal of a dynamic pressure sensor (left) and a fiber optical probe (right) filtered for $\mathrm{OH}^{*}$ flame emission of a previous BKD test campaign [8].

resonance modes of the LOX injectors. As can be seen in Fig. 1 the dominant $\mathrm{OH}^{*}$ lines are present for both stable and unstable conditions. For that reason Gröning concluded that the injectors have a more independent behavior [10]. The coupling mechanism was therefore described as injection-driven: the instabilities occur when the frequency of the chamber 1T mode matches to the second longitudinal mode of the LOX post [8].

A connection of LOX post acoustics to the driving of combustion instabilities is a phenomenon not specifically associated with the BKD design, but has also been reported for other LOX/H2 experiments $[6,11]$ and even full-scale main stage engines as the $\mathrm{J}$ 2S [4] and the recent Japanese LE-X [7]. Also for the propellant combination LOX/CH4 examples of LOX injection-coupling can be found $[12,13]$. So it seems that the LOX post acoustics play a significant role in the coupling mechanism leading to combustion instability for cryogenic rocket engines and gaining a better understanding of the detailed processes may help to prevent this kind of thermoacoustic driving for future LPREs. In previous BKD investigations fiber optical probes helped to find the basic coupling mechanism. However, there still remained unsolved questions as how the flame reacts to the LOX injector oscillation and how the energy is transferred from the LOX post acoustics, 
through the fluctuating flame into the acoustic field of the chamber. To address the given questions it was decided to conduct new test campaigns with a challenging design with optical access into BKD allowing a two-dimensional (2D) flame visualization.

The goal of this study is to investigate the 2D flame dynamics. It is analyzed how the flame actually behaves at the LOX injector frequencies. Besides, the flame dynamics are compared for stable and unstable conditions, because with another DLR research combustor with acoustic forcing it was observed that the flame is strongly influenced by acoustic oscillations of the chamber modes $[14,15]$.

\section{Experimental setup}

\subsection{Thrust chamber $B K D$}

The hot-fire tests for this analysis were conducted with the multi-element DLR research thrust chamber $D(B K D)$. During operation self-excited high-frequency combustion instabilities appeared for certain operating conditions. Therefore BKD offers a valuable experimental setup to analyze the underlying coupling mechanism under representative conditions, namely a cylindrical chamber cross section, a multi-element injector head, the cryogenic propellant combination LOX/H2 and chamber pressures in the supercritical regime with respect to the injected propellants oxygen and hydrogen.

The thrust chamber as presented in Fig. 2 consists of the main components injector head, HF measurement ring, a $200 \mathrm{~mm}$ long cylindrical chamber segment and a convergent-divergent nozzle.
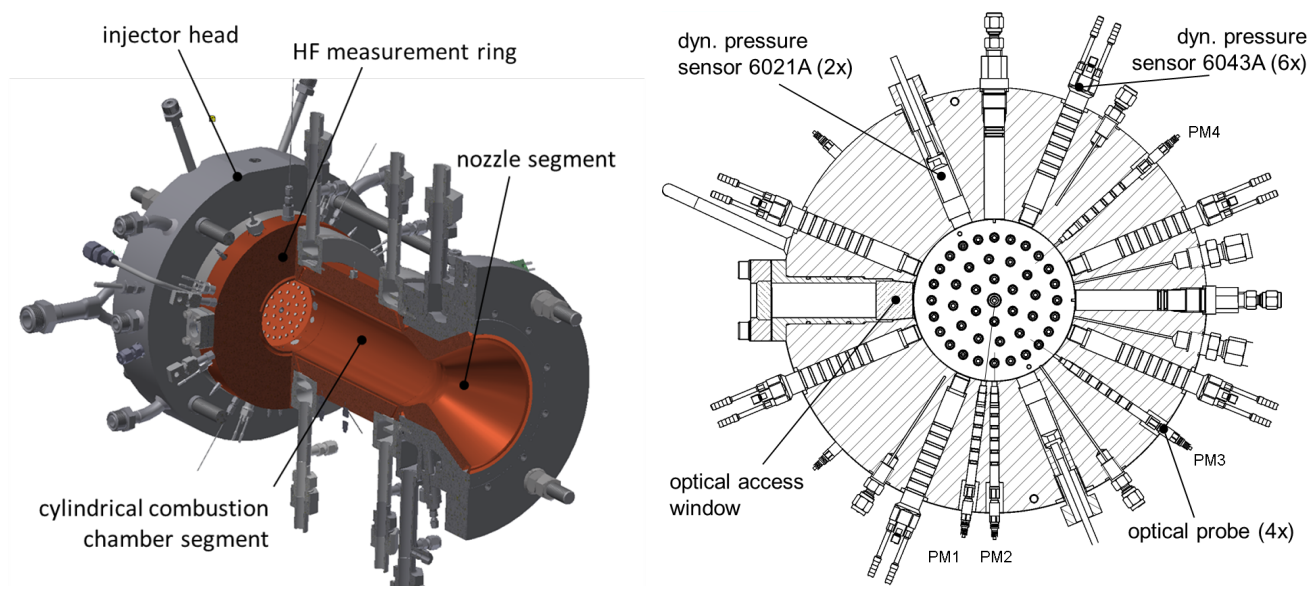

FIGURE 2. Thrust chamber BKD (left) with optical access ring (right).

In order to achieve optical access into the combustion chamber, a new water-cooled HF measurement ring has been designed and replaced the previously used ring $[8,16]$. For this reason the characteristic chamber length [2] increased slightly from $L^{*}=0.64 \mathrm{~m}$ to $L^{*}=0.66 \mathrm{~m}$. More details about the optical access can be found in section 2.3. Since the conditions in BKD are very harsh, also a local film cooling injection slot in front of the optical access window with a height of $0.45 \mathrm{~mm}$ was added for the recent test campaigns. The propellants are injected through 42 shear coaxial injection elements with a recessed and tapered LOX post. The inner diameter of the LOX posts is $3.6 \mathrm{~mm}$. The length of the recess is $2 \mathrm{~mm}$, or $0.54 d_{o x}$. 


\subsection{Operating conditions}

In past campaigns, a large number of different load points were realized in one test [8]. However, to reduce the risk of window failure in the recent campaign BKD-OPT-17, the test duration and amount of load points were reduced. A load point is hereby mostly defined by the static combustion chamber pressure $p_{c c}$, which is in BKD typically between 50 and 80 bar, and the propellant mixture ratio $\left(R O F=\dot{m}_{O_{2}} / \dot{m}_{H_{2}}\right)$ from 2 to 6.8 . For the most extreme load point of $p_{c c}=80$ bar and ROF $=6$ a thrust of about $24 \mathrm{kN}$ can be realized, which makes BKD comparable to small upper-stage engines. Images of a test run with optical access at the test bench P8 are presented in Fig. 3. In order to purge and cool the sapphire window, a small amount of hydrogen, from 0.6 to $0.7 \%$ of the total chamber mass flow, is used. For this purpose the test bench provides hydrogen at ambient temperature. The additional film cooling hydrogen slightly reduces the bulk ROF in the chamber by about $5 \%$. A typical test sequence and the corresponding spectrogram of a chamber dynamic pressure sensor is presented in Fig. 4.

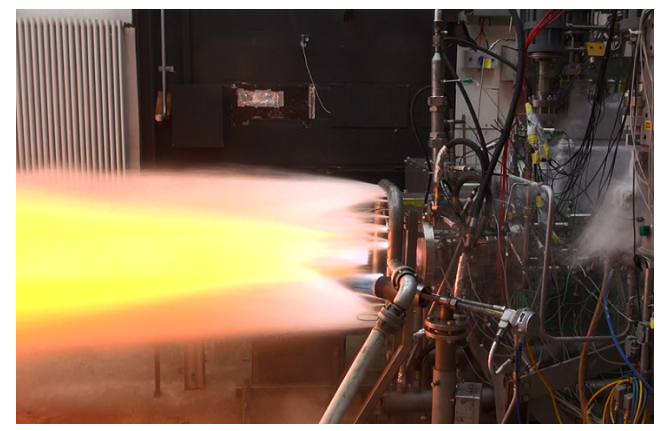

FIGURE 3. Hot-fire test of BKD with optical access at the test bench P8
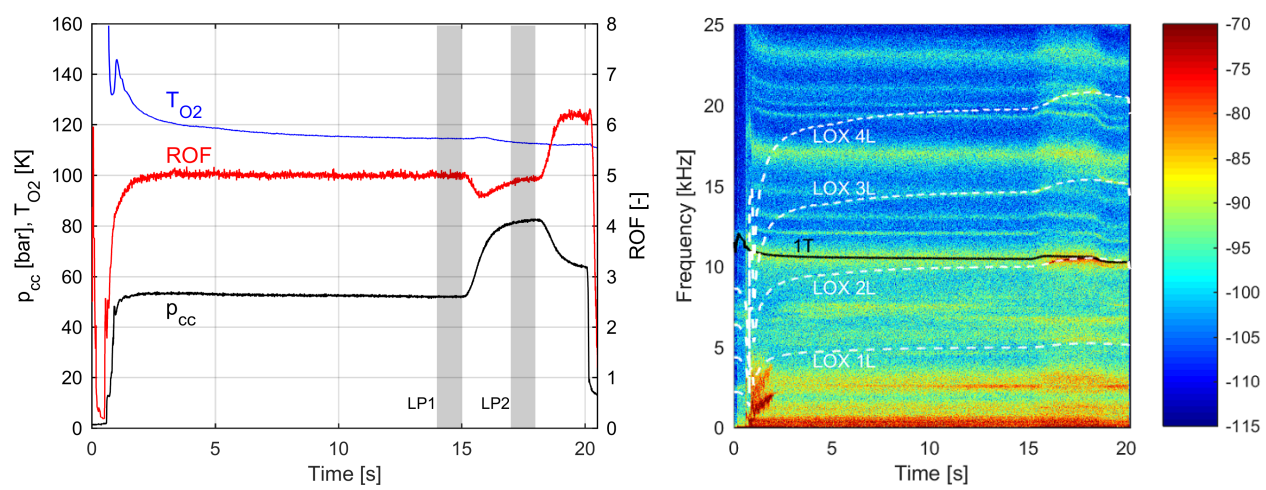

FIGURE 4. Left: Test sequence of a BKD test run with optical diagnostics. Right: corresponding spectrogram of dynamic chamber pressure.

In the spectrogram the $1 \mathrm{~T}$ mode frequency is indicated with a black line. The LOX post acoustic mode lines are also presented according to the measured wave numbers [8]. Two different load points have been selected for the analysis of the high-speed flame imaging data. Based on the knowledge of the basic coupling mechanism and the influence of operating conditions on the acoustic field of the chamber, as described by 
Gröning et al. $[8,10]$, a load point with strong flame-acoustic interaction and with low interaction respectively, were chosen. The main operating parameters of the two load points are defined in Table 1.

$\begin{array}{lll}\text { Load point } & \text { LP1 (low coupling) } & \text { LP2 (strong coupling) } \\ p_{c c}[\mathrm{bar}] & 51.8 & 81.7 \\ R O F[-] & 5 & 4.92 \\ R O F_{\text {bulk }}[-] & 4.7 & 4.75 \\ T_{O 2}[\mathrm{~K}] & 114.4 & 112.7 \\ T_{H 2}[\mathrm{~K}] & 102.5 & 103.4\end{array}$

TABLE 1. Operating conditions

\subsection{Optical diagnostics}

In previous BKD instability research campaigns fiber-optical probes were used to investigate the coupling mechanism. The probes contain a sapphire rod and the flame radiation is transferred to Photomultipliers (PM) by optical fibres. Interference filters for the $\mathrm{OH}^{*}$ radiation with a center wavelength of $310 \mathrm{~nm}$ and a full width at half-maximum of $10 \mathrm{~nm}$ are mounted in front of the PMs. The PM signals are also recorded with $100 \mathrm{kHz}$. The full acceptance angle of the probes is less than $2^{\circ}$. This feature has been used to align the optical probes to specific injectors [8]. Four probes are mounted in the new ring at the measurement plane $5.5 \mathrm{~mm}$ downstream of the faceplate, as indicated with the thin lines in Fig. 2.

The new water-cooled measurement ring for BKD, allows a small optical access sapphire window with a diameter of $18 \mathrm{~mm}$ to be installed. The window is positioned to be centrally aligned with one injector flame and to have a slight overlap to the faceplate, so that the full injection diameter is visible at the injection plane. The length or diameter of the window was limited by the rapidly increasing thermal loads downstream of the injection plane. Nevertheless, numeric unsteady CFD simulations of BKD indicated that this region close to the faceplate is of major importance for the thermoacoustic coupling. The URANS modelling of a single element flame by Schulze et al. [17] came to the result, that about $45 \%$ of the total Rayleigh integral driving happens in the first $15 \mathrm{~mm}$ of the combustion chamber. LES simulation by Urbano et al. [18] yielded similar results.

For the propellant combination oxygen-hydrogen flame visualization is usually made with $\mathrm{OH}^{*}$ chemiluminescence in the UV range at around $308 \mathrm{~nm}$. High-speed flame radiation imaging has also widely been used to study acoustic-flame interaction in rocket engines [11,19-22]. However, recent analysis of the flame emission spectra of hydrogenoxygen flames at different pressure levels by Fiala et al. revealed some issues of $\mathrm{OH}^{*}$ radiation at the extreme conditions of LPREs, as strong self-absorption and thermal excitation [23-25]. On the other hand Fiala et al. also investigated the blue radiation of oxygen-hydrogen flames $[24,25]$, which is in the visible range with a broad peak showing highest intensity in the blue wavelength between $420 \mathrm{~nm}$ [26] and around $440 \mathrm{~nm}$ [25]. The blue radiation does not suffer from strong self-absorption and therefore allows the flame dynamics to be analyzed with a greater depth of field than $\mathrm{OH}^{*}$. The experimental investigation of broadband visible radiation in another DLR combustor, called $\mathrm{BKH}$, by Webster et al. showed that this alternative to the well-known $\mathrm{OH}^{*}$ emission, is well suited to observe flame dynamics with increased depth of view at high pressure conditions [20]. 
Simultaneous and synchronized high-speed flame radiation measurements of $\mathrm{OH}^{*}$ $(310 \pm 5 \mathrm{~nm})$ and blue $(436 \pm 5 \mathrm{~nm})$ radiation were realized in a recently conducted test campaign. An illustration of the optical diagnostic setup can be found in Fig. 5. The Photron Fastcam high-speed cameras allowed to reach an acquisition rate of 60,000 frames per second (FPS), resulting in 6 images per instability period. Both cameras were syncronized in a Master-Slave setup. The resolution for both cameras was about $110 \times 110$ pixels and the monochrome intensity resolution was 12-bit grayscale.

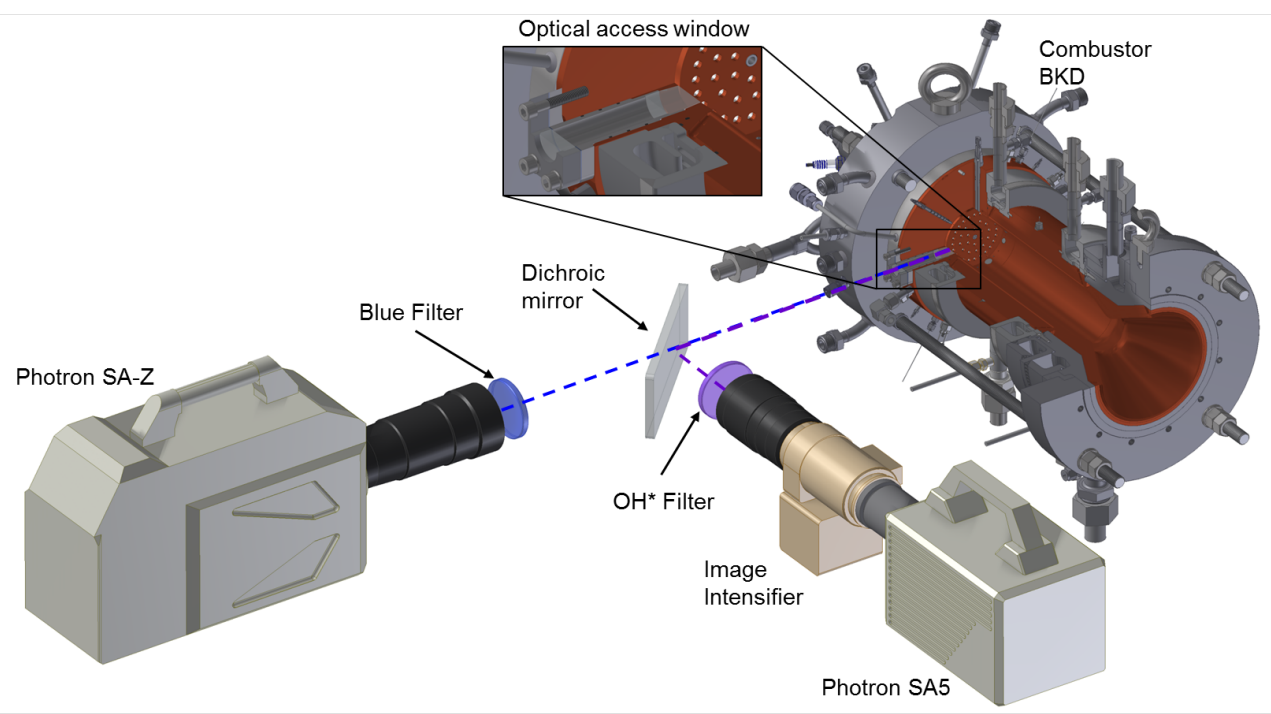

FIGURE 5. Optical diagnostics setup.

\section{Methodology}

\subsection{Dynamic mode decomposition}

The main focus of this analysis is to investigate the flame dynamics at certain frequencies. For that purpose dynamic mode decomposition (DMD) was applied to the highspeed flame radiation measurements. DMD as described by Schmid et al. [27] enables the extraction of dominant periodically fluctuating phenomena at different frequencies. In the past, the DMD method has been used in thermoacoustic instability research in order to extract the distributed flame response at certain frequencies from flame visualization data $[15,19,28,29]$.

Both the $\mathrm{OH}^{*}$ and blue flame radiation data were decomposed in their dominant periodic modes by DMD. Sequences of 6000 images were processed. With a sampling rate of 60,000 FPS, the corresponding frequency resolution of the DMD modes is $10 \mathrm{~Hz}$. Each DMD mode is thereby defined by a temporal component, the temporal mode, that describes how the mode oscillates in time and a spatial mode including the information of the intensity fluctuation of each pixel around the mean value. 


\section{Results and Discussion}

\subsection{Acoustic field characteristics}

As described before, the test length has been reduced compared to previous test campaigns. So the test run presented in Fig. 4 has a relatively long duration with a chamber pressure of 50 bar (LP1), before changing to a more harsh condition of 80 bar and a bulk ROF of about 4.8 with a close frequency spacing between LOX post $2 \mathrm{~L}$ mode and the chamber 1T mode, leading to an excitation of the chamber 1T mode. Fig. 6 shows the pressure field characteristics for the analyzed BKD test run with optical access presented in Fig. 4.
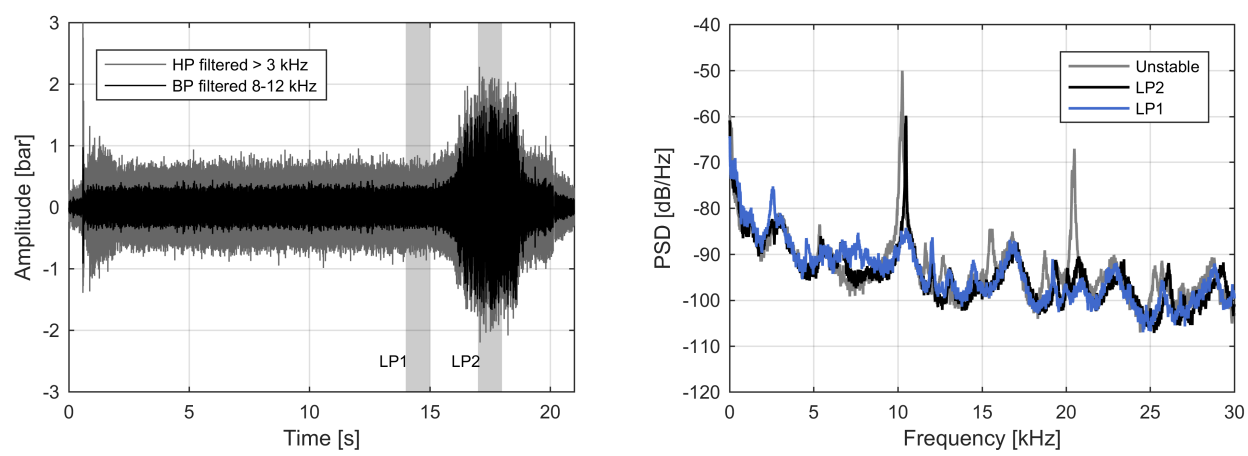

FIGURE 6. Pressure field characteristics for the test run with optical access. Left: bandpass and high-pass filtered dynamic pressure signal of the test shown in Fig. 4. Right: PSDs of the two load points with low and strong coupling in comparison to the classic unstable load point in Fig. 1.

The left hand side of Fig. 6 shows high-pass filtered and 1T-bandpass filtered dynamic pressure sensor signals. A self-excited 1T mode can be observed for the load point 2 (LP2) at the 80 bar chamber pressure stage. High-pass filtered peak-to-peak amplitudes for LP2 are 4.5 bar, or $5.5 \%$ of $p_{c c}$ and are therefore on the edge to be called unstable [2, 10]. The right hand side presents a comparison of power spectral densities (PSDs) for the dynamic chamber pressure. The PSDs were calculated over $1 \mathrm{~s}$ using the Welch method and Hanning windows with a length of 8192 samples and a $50 \%$ overlap. The reference pressure for the $\mathrm{dB}$ scaling was 80 bar, the maximum chamber pressure of BKD. The blue PSD was calculated for the load point with low coupling (LP1) and shows the typcial acoustic spectrum for stable operation of BKD $[8,16]$. The gray PSD shows the well-known type 1 instability of BKD, which is presented in Fig. 1 and is calculated for that test run for the time window of 24-25 s. The sharp peak of the 1T mode around $10 \mathrm{kHz}$ can clearly be observed. Due to the high $1 \mathrm{~T}$ amplitudes of the unstable load point, the first overtone resembling the pressure distribution of the first radial chamber mode can be detected at $20 \mathrm{kHz}$. The black curve is calculated for LP2, the load point of the optical access test run with strongest coupling. A sharp peak of the 1T mode can also be observed at a slightly higher frequency of $10.47 \mathrm{kHz}$. The peak height decrease of $10 \mathrm{~dB}$ indicates a reduction of $1 \mathrm{~T}$ amplitude reaching about $30 \%$ of the amplitudes in early BKD campaigns. Nevertheless, Fig. 4 and Fig. 6 still show that general behavior is similar to the previously published coupling mechanism [8]: as soon as the chamber 1T mode frequency approaches the $2 \mathrm{~L}$ mode of the LOX post, the chamber $1 \mathrm{~T}$ mode gets excited by the oscillating flame and dominates the chamber acoustics 


\subsection{Flame phenomenology}

At first, exemplary instantaneous images and mean images of both the $\mathrm{OH}^{*}$ and the blue high-speed camera are presented for the low coupling load point LP1 in Fig. 7. For this load point the static combustion chamber pressure was about 50 bar, so the injection conditions are transcritical with respect to the critical pressure of oxygen. An exemplary single, instantaneous image for both wavelengths is shown in the upper row. The two different flame radiation diagnostics show the same, synchronized frame. The mean images averaged over 6000 frames, hence a duration of $0.1 \mathrm{~s}$, are shown below.
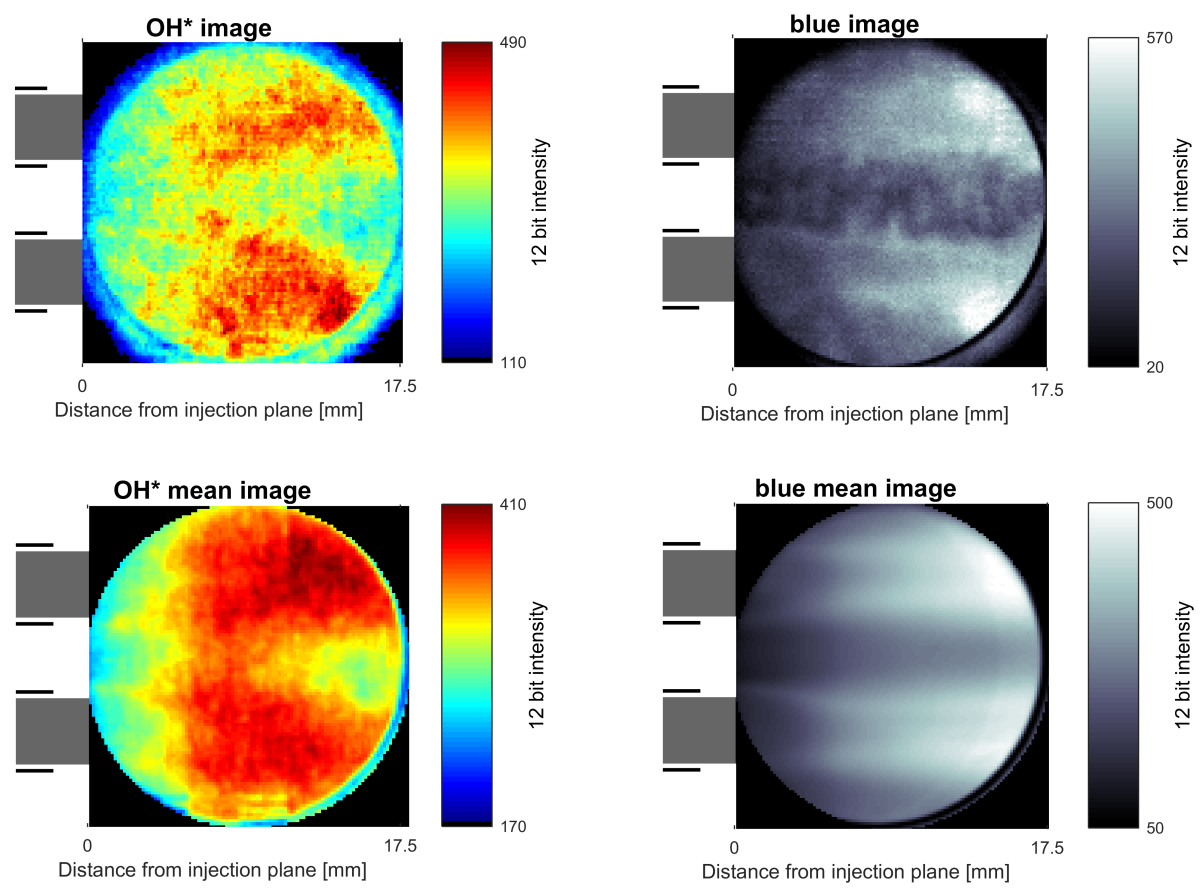

FIGURE 7. Instantaneous and mean flame radiation images for LP1. Left: $\mathrm{OH}^{*}$ emission in the UV. Right: blue radiation.

In the $\mathrm{OH}^{*}$ images the thin shear layer of the developing flame can be detected at the left side of the images, starting from the faceplate. The flame diameter is then rapidly increasing, until at about one third of the window length, so approximately 1.6 times the LOX post diameter, the converging high radiation intensity regions of the central flame merge with the neighboring flames. This behavior can mainly be observed in the $\mathrm{OH}^{*}$ mean image, where after $1 / 3$ of the window length the downstream area of the window is mostly filled with high intensity. However, in the last downstream third a region with lower intensity can be observed in both the instantaneous and mean image of $\mathrm{OH}^{*}$ radiation. This low intensity spot was not expected, because it is placed on top of the central flame, where one would expect strongest intensity.

The blue radiation shows more details than $\mathrm{OH}^{*}$. Both the single shot as well as the mean image show the central dense LOX core as a dark jet. The combustion zone around the dark LOX core can also be observed as a thin area of high intensity. Parts of the two neighboring flames can also be seen at the top and bottom of the window. 
In general the observations fit to the results of Webster et al. [20], who reported a comparable overall flame structure between $\mathrm{OH}^{*}$ and visible imaging, but with higher optical depth in the visible wavelengths.

The instantaneous blue radiation image shows that the processes inside BKD, as LOX injection, atomization and combustion are highly turbulent and influenced by combustion noise even for stable combustion conditions. The dense LOX core is not smooth but shows many small scale structures, which in this case seem to be rather random. The mean image is in agreement with $\mathrm{OH}^{*}$, the high intensity zones of the individual flames are diverging and come close to the neighboring flame after about $1 / 3$ of the window length. At the downstream end of the window the three flames can hardly be distinguished due to the merging combustion zones, which shows that in multi-element combustion chambers with a dense injector pattern flame-flame interaction can have an impact on atomization and combustion after less than three injector diameters and should not be excluded in combustion modelling.

Based on the combined impression from the images in Fig. 7, a hypothesis may be offered to explain the spot of low intensity in the $\mathrm{OH}^{*}$ images. In the blue imaging with greater depth of field, the increasing thickness of the flame in the direction of the flow can be seen yet there is little change in intensity of the form of the LOX core at the centre of the image. Therefore the low intensity spot in $\mathrm{OH}^{*}$ images may be the result of a weakening of the $\mathrm{OH}^{*}$ radiation source - the reaction zone - between the LOX core and the window. The spreading flame may come into contact with the window cooling film flow here, flattening and thinning it. A CFD simulation would likely assist in the interpretation of this feature.

Figure. 8 shows images for LP2. As for LP1, interpretation of the instantaneous $\mathrm{OH}^{*}$ image in Fig. 8 is difficult. The averaged $\mathrm{OH}^{*}$ image shows similar characteristics as for the LP1 case. In the first third of the window the developing shear layer of the central flame can be observed, until the high intensity zone merges with the neighboring flames. Also present here is the low intensity spot at the downstream end of the window, for which there is no indication in the blue images Figs. 8.

The flame phenomenology in the blue radiation imaging is similar to LP1, but the flames don't seem to widen up as rapidly as in the 50 bar case of LP1. The thin combustion zone shear layers interact with neighboring flames a bit later at about half of the window diameter. This is most likely due to the higher impulse of the injected propellants. In the instantaneous blue image small scale wave-like structures on the dense LOX jet can be detected. In comparison to LP1 the structures seem to be stronger periodically organized, which could be a first indication of the 2D flame response to the LOX injector fluctuation.

\subsubsection{Flame dynamics for high thermoacoustic interaction}

A DMD analysis has also been conducted for the low coupling load point LP1. Nevertheless, no dominant DMD modes at the resonance frequencies of the LOX injectors could be found for both the $\mathrm{OH}^{*}$ and the blue camera. Therefore only the results for LP2 are presented here.

Fig. 9 shows the DMD mode energies of both cameras for the second load point with stronger coupling. The ranges of the oxygen injector eigenfrequencies based on experimentally measured wave numbers [8] are indicated with highlighted background in gray. Now flame response to the injector acoustics, $1 \mathrm{~L}$ around $5 \mathrm{kHz}$ and $2 \mathrm{~L}$ at $10 \mathrm{kHz}$ can be observed in blue and $\mathrm{OH}^{*}$ radiation. For the $3 \mathrm{~L}$ LOX post mode a flame response can not be found with the DMD method, but just above $20 \mathrm{kHz}$ a peak can be detected 

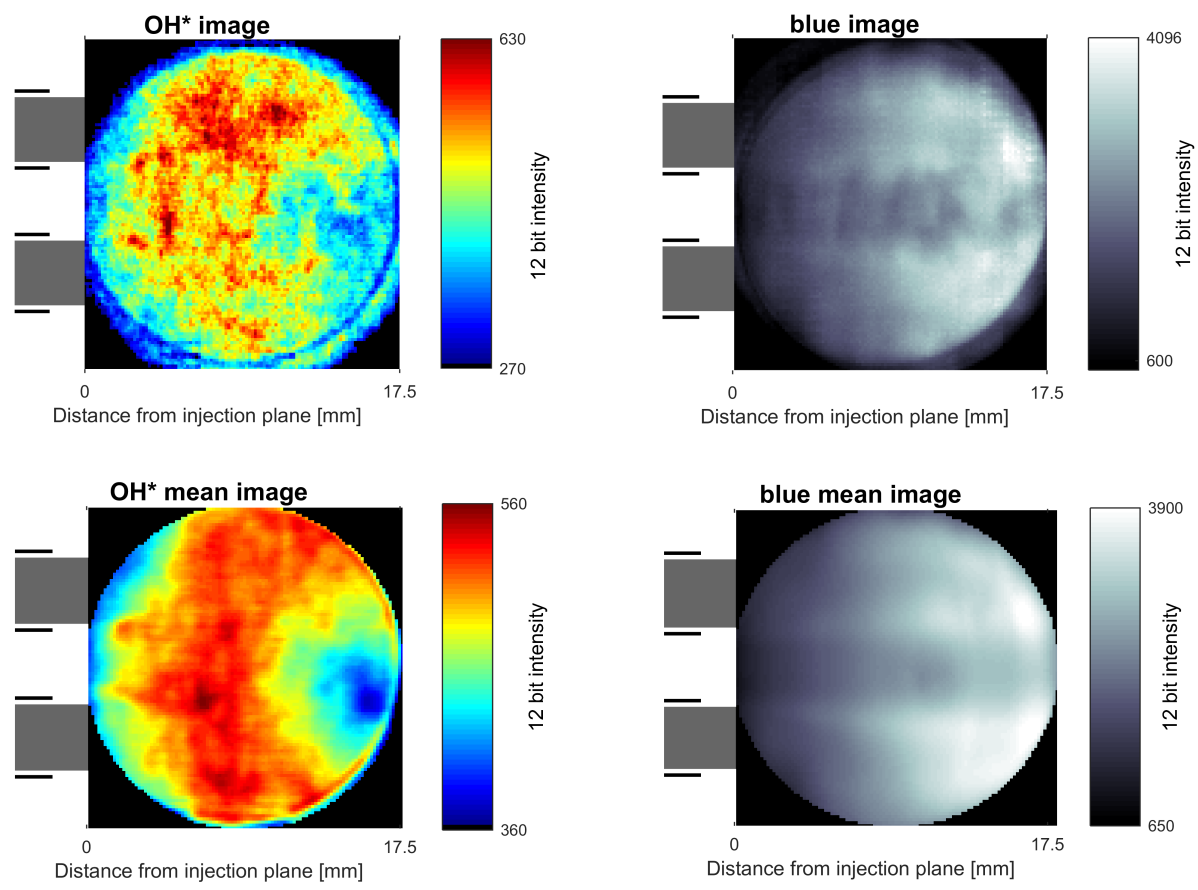

FIGURE 8. Instantaneous and mean flame radiation images for LP2 with strong coupling. Left: $\mathrm{OH}^{*}$ emission in the UV. Right: blue radiation.

which should correspond to the fourth injector mode. Nevertheless, in the following it will be focused on the flame response to the fundamental injector mode (1L) and the first overtone $(2 \mathrm{~L})$, because of the coupling with the chamber $1 \mathrm{~T}$ mode. Therefore a more detailed view of that frequency range is presented on the right side of Fig. 9. The sharp peak at $10.5 \mathrm{kHz}$, was identified as the chamber 1T mode, based on comparison with the acoustic spectra. The rather broad peak below the 1T mode shows the flame response to the $2 \mathrm{~L}$ LOX post mode. For that reason Fig. 15 allows to clearly distinguish between the combustion chamber acoustics (1T mode) and the LOX post acoustics, which depicts that a perfect frequency match between LOX post $2 \mathrm{~L}$ and chamber $1 \mathrm{~T}$ mode was not achieved. The analysis of Gröning et al. showed that high 1T amplitudes are only experienced for a narrow frequency spacing [8] and therefore Fig. 9 could also explain, why in the given case a lower 1T amplitude was observed.

In order to investigate the detailed flame response to the LOX injector oscillation, the DMD modes with the highest magnitude, hence the most coherent structures, within the frequency ranges of LOX injector $1 \mathrm{~L}$ and $2 \mathrm{~L}$ eigenmodes, have been added to form a dynamic flame response. The resulting $2 \mathrm{D}$ flame response to the LOX post $1 \mathrm{~L}$ mode combined of 10 individual DMD modes is presented in Fig. 10. It can be observed that the flame reacts periodically with an axisymmetric intensity distribution indicating small scale structures with several wave lengths, about 3-4, in the visible area of the optical access window. Regarding the longitudinal distribution for the $1 \mathrm{~L}$ mode, both wavelengths show a similar shape for the flame response. However, as in the mean images the DMD results of the blue wavelengths contains more details.

The combined flame response to the $2 \mathrm{~L}$ injector oscillation is shown in Fig. 11. For the 

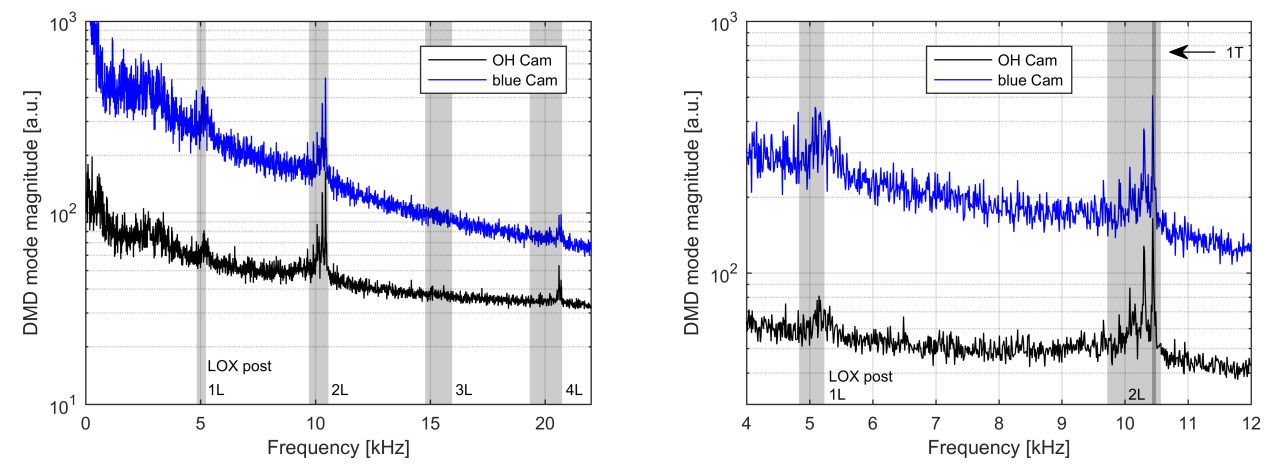

FIGURE 9. DMD mode magnitude over frequency for the strong coupling load point (LP2). A more detailed view of the relevant frequency range is shown on the right hand side. The $1 \mathrm{~T}$ frequency for this load point extracted from the p' PSD is also indicated.

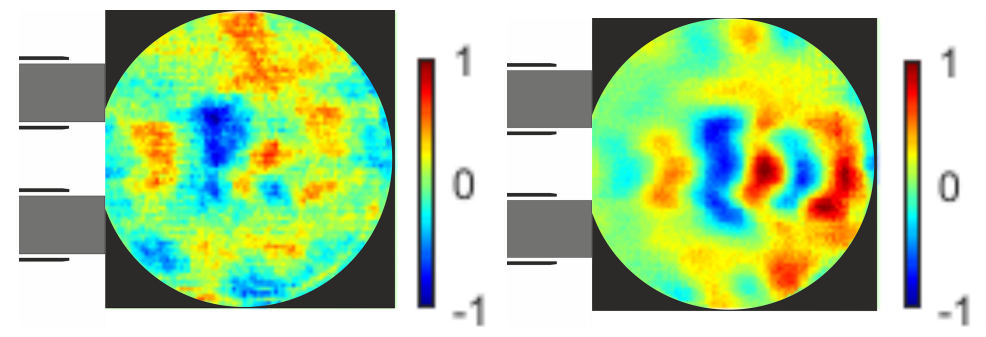

FIGURE 10. Spatial distribution of flame dynamics in the window region for the chosen DMD modes at the first longitudinal $(1 \mathrm{~L})$ resonance modes of the LOX post. Left: $\mathrm{OH}^{\star}$. Right: blue.

$2 \mathrm{~L}$ case $10 \mathrm{DMD}$ modes for the blue wavelengths and $12 \mathrm{DMD}$ modes for $\mathrm{OH}^{*}$ respectively, were merged to form the flame response images. For the $2 \mathrm{~L}$ mode these modes included also the $1 \mathrm{~T}$ mode with a rather homogeneous flame radiation intensity fluctuation. Therefore the flame response to the second longitudinal mode is still visible in the center of the window with a longitudinal periodic and almost symmetric distribution, but more disturbance due to the chamber $1 \mathrm{~T}$ mode oscillation on the flame structure can be observed. Also in the case of the $2 \mathrm{~L}$ flame response, there is a larger discrepancy between $\mathrm{OH}^{*}$ and the blue radiation, which is most likely due to the greater depth of view for the blue radiation. Therefore it allows to still see small scale structures on the dense LOX jet corresponding to the 2L LOX post mode, whereas the $\mathrm{OH}^{*}$ image shows the intensity fluctuation closer to the window.

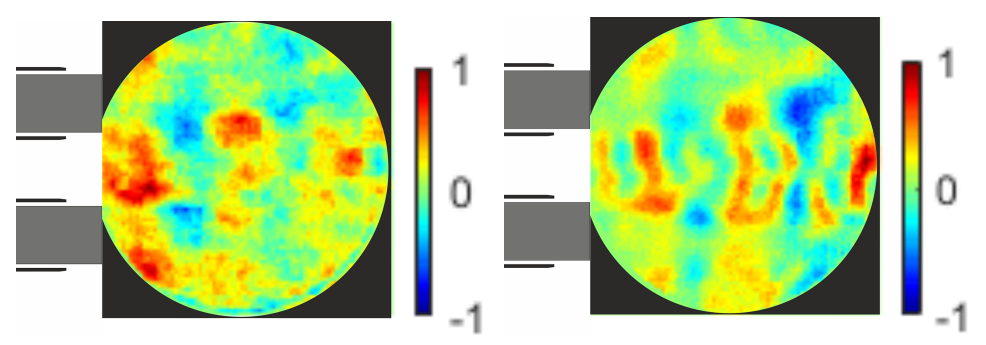

FIGURE 11. Spatial distribution of flame dynamics in the window region for the chosen DMD modes at the second longitudinal $(2 \mathrm{~L})$ resonance modes of the LOX post. Left: $\mathrm{OH}^{\star}$. Right: blue. 
In summary, the analyzed flame response at around $5 \mathrm{kHz}$ and $10 \mathrm{kHz}$ was calculated by DMD and showed a longitudinal and almost symmetrical shape. This matches to the observations of Gröning et al. [8], describing that these dominant flame oscillation frequencies are generated by acoustic resonance modes of the LOX injectors. In addition, the DMD analysis showed a highly resolved mode magnitude distribution for the $\mathrm{OH}^{*}$ and blue radiation, which allowed to distinguish between the chamber $1 \mathrm{~T}$ mode with a rather homogeneous intensity fluctuation and the flame response to the injector modes. The frequency spacing between chamber $1 \mathrm{~T}$ mode and the second LOX injector flame response in the DMD result was larger than expected, which explains why in the analyzed test run, the observed 1T amplitude was lower as for previously published BKD combustion instability investigations.

\subsubsection{Preliminary analysis of LOX post acoustics source}

In previous BKD test campaigns it was demonstrated that the flames in BKD oscillate with acoustic resonance frequencies of the LOX posts. Nevertheless, the source of the LOX post acoustics is still unknown. Gröning et al. [8] described that the dominant $\mathrm{OH}^{*}$ lines of the flame are also present for stable combustion conditions. For this reason the authors concluded that the instability mechanism is not a fully closed loop in which a chamber 1T mode oscillation excites the injector acoustics which would lead to the oscillating flames that further excite the $1 \mathrm{~T}$ amplitude, but rather permanently oscillating flames, which can excite the 1T mode for a close frequency spacing. For this rather independent behavior of the LOX injectors, another acoustic excitation source needs to be found. The two most presumable sources are general turbulent broadband combustion noise from the chamber, or hydrodynamic effects in the injector head itself [10].

In contrast to the previous BKD observations, in the current study no flame response peaks at the resonance frequencies of the LOX posts were observed for the 50 bar case LP1. So, a better understanding of the LOX post excitation source could also help to explain the different behavior for the two load points.

In order to achieve a more homogeneous LOX injection over the 42 injectors, a sharp edged inlet throttle orifice is mounted between the injector head manifold and the LOX post tubes. A common phenomenon of orifice flow is a so called whistling orifice, sound generated by hydrodynamic effects as vortex shedding and turbulence. A nondimensional relation to analyze vortex shedding frequencies is given by the Strouhal number. For whistling orifice flows the Strouhal number is given by Eq. 4.1, with the throttle thickness $t$, the whistling frequency $f$ and the flow velocity in the orifice $u_{\text {throttle }}$. Typical Strouhal number values for orifice flow hydrodynamics exciting the tube acoustic eigenmodes are in the range 0.2-0.35 [30]. Hitt et al. found a maximum response for the flow of cryogenic nitrogen through a thin orifice at a Strouhal number of $0.28 \pm 10 \%$ [31]. The investigation of Lacombe et al. [32] came to the result that acoustic amplification due to orifice whistling can be observed in the range 0.2 to 0.4 .

$$
S t=\frac{f \cdot t}{u_{\text {throttle }}}
$$

Usually measured pressure or flow oscillation PSDs are used to calculate the nondimensional Strouhal number frequency spectra. Nevertheless, the BKD design does not allow dynamic pressure oscillation measurements in the LOX posts themselves and therefore $\mathrm{OH}^{*}$ oscillation measurements by the optical probes, which are known to show the LOX post resonance frequencies as dominant lines [8], have been used for the analysis. Since the intensity oscillation corresponding to the $2 \mathrm{~L}$ mode of the LOX post is 
strongly depending on the coupling to the chamber $1 \mathrm{~T}$ mode, the first LOX post mode is analyzed. Fig. 12 shows PSDs of the normalized $\mathrm{OH}^{*}$ intensity of the optical probes plotted over the calculated Strouhal number for both load points. For the low coupling condition shown on the left hand side the theoretical range of the LOX post $1 \mathrm{~L}$ mode according to experimental wave numbers [8] yields Strouhal numbers of about 0.5-0.54, which is above the potential hydrodynamic excitation values. In addition as described before, no dominant $\mathrm{OH}^{*}$ oscillation peaks corresponding to the LOX post mode can be found for this load point. For LP2 the same analysis was conducted and is presented in the right plot of Fig. 12. For this load point dominant peaks are present which match to the $1 \mathrm{~L}$ mode frequency range, but most importantly the frequency of the $1 \mathrm{~L}$ mode of the post yields in this case Strouhal numbers around 0.35 , which is in the range where acoustic excitation due to orifice whistling phenomena have been described in literature [30-32]. Therefore it is possible that hydrodynamic effects as periodic vortex shedding at the LOX post throttle orifice excites the LOX posts or at least may amplify the acoustic oscillations in the LOX post. The whistling orifice effect would also explain, why in the DMD analysis and the Photomultiplier spectral analysis no LOX injector peaks were found.
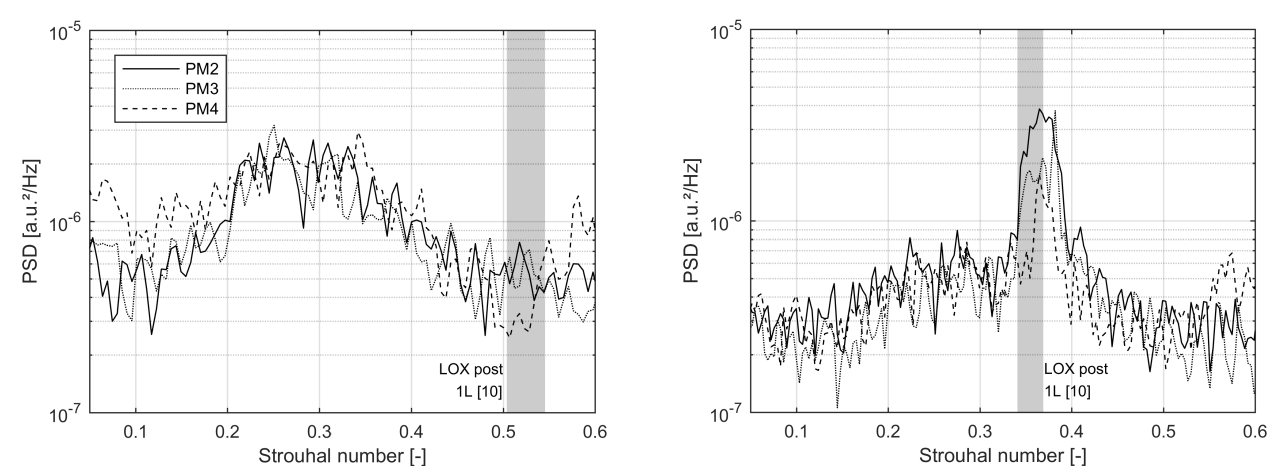

FIGURE 12. PSDs of normalized $\mathrm{PM} \mathrm{OH}^{*}$ intensity fluctuation around the $1 \mathrm{~L}$ mode of the LOX posts plotted over the Strouhal number. Left: LP1, right: LP2.

However, in order to further underline the hydrodynamic LOX post acoustic amplification, the test run presented in Fig. 1 including a large number of operating conditions was also analyzed. By closely looking at Fig. 1, it can be detected that the dominant $\mathrm{OH}^{*}$ lines in the PM spectrogram on the right are mostly present for stable and unstable conditions at increased chamber pressures, whereas for the 60 bar stage the lines get weaker. In order to investigate the complete test run including a large number of different operating conditions, the test was divided into $0.2 \mathrm{~s}$ long time windows. For each window the bandpass-filtered and normalized $\mathrm{OH}^{*}$ oscillation at the $1 \mathrm{~L}$ frequency $\left(I_{1 L}^{\prime} / \bar{I}\right)$ was calculated and plotted over the Strouhal number of the LOX post $1 \mathrm{~L}$ peak frequency. The results are presented in Fig. 13 and match to the hypothesis. The strongest $\mathrm{OH}^{*}$ oscillation at the first longitudinal LOX injector frequency can be observed for orifice Strouhal numbers around 0.35, whereas for increasing Strouhal numbers, the $\mathrm{OH}^{*}$ flame response to the LOX post $1 \mathrm{~L}$ mode decreases rapidly. In addition, the chamber pressure of each time window is shown by the gray scale of the markers and it can be seen that the Strouhal number, hence the injector $1 \mathrm{~L}$ mode flame oscillation, is defined by the combustion chamber pressure $p_{c c}$. This observations further underlines 
that the injectors have a rather independent behavior and are not mainly influenced by chamber pressure oscillations, because for 80 bar chamber pressure both stable and unstable conditions were observed in this particular test run, but most of the 80 bar points show an increased $\mathrm{OH}^{*}$ oscillation for the $1 \mathrm{~L}$ LOX injector frequency. Also for the 70 bar stage Gröning defined the ROF 6 load point as semi-unstable [10], but all 70 bar points in Fig. 13 still show lower $\mathrm{OH}^{*}$ oscillation due to the lower Strouhal numbers.

In summary, the presented results give a strong indication that hydrodynamic effects at the LOX post throttle play an important role in the acoustic excitation of the LOX posts. This would also explain why in past campaigns also for stable combustion cases dominant $\mathrm{OH}^{*}$ lines were observed and why for the 50 bar stage in this study, the flame oscillation by the LOX post acoustics did almost disappear. However, the analysis of the Strouhal-related hydrodynamic excitation has so far only been analyzed for two test runs and further investigation is necessary in the future.

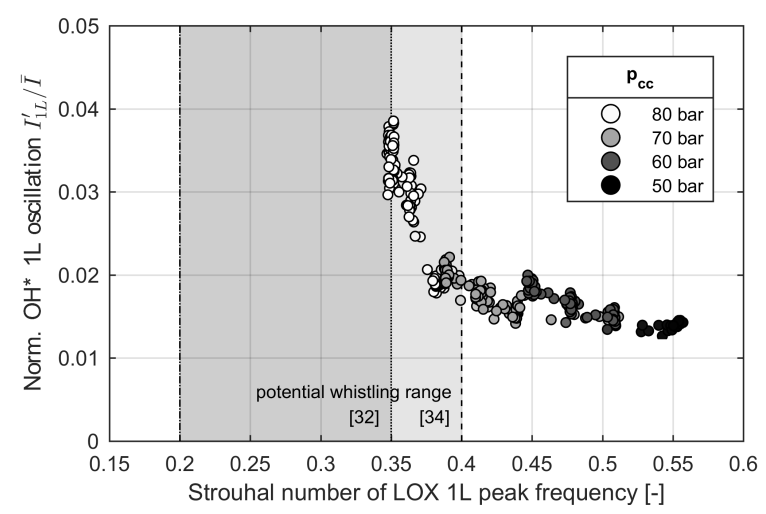

FIGURE 13. Normalized $\mathrm{OH}^{*}$ intensity fluctuation at LOX $1 \mathrm{~L}$ frequency plotted over $1 \mathrm{~L}$ peak Strouhal number for $0.2 \mathrm{~s}$ time windows of the run presented in Fig. 1

\section{Summary and Conclusions}

The DLR research thrust chamber BKD shows self-excited combustion instabilities under representative conditions. From previous work, the coupling mechanism is known to be related to LOX injector acoustics. A new optical access window was implemented to observe the flame reaction to LOX injector oscillations. With the window it was possible to realize 2D flame visualization for highly representative engine conditions, including static combustion chamber pressures of up to 82 bar and bulk propellant mixture ratios up to 6.6. High-speed imaging in the blue and $\mathrm{OH}^{*}$ radiation wavelengths with a frame rate of 60,000 FPS was used to study the acoustic-flame interaction for one of the 42 injectors.

Two different load points were chosen to study the flame phenomenology and the flame dynamics, one with low flame-acoustic interaction at a static chamber pressure of 50 bar and the second load point with stronger thermoacoustic coupling at 80 bar $p_{c c}$. The first load point is close to the critical pressure of oxygen. Therefore large variations of the oxygen fluid properties can occur in the chamber, which could also impact the thermoacoustic coupling. Nevertheless, in the area of the optical access window close to the injection plane, a similar general flame phenomenology to LP2 could be observed 
in both the $\mathrm{OH}^{*}$ and blue imaging. In general the blue flame radiation imaging showed more details than the well-known $\mathrm{OH}^{*}$ emission.

The flame dynamics were investigated with dynamic mode decomposition (DMD) of the $2 \mathrm{D}$ imaging. For the first load point no dominant $\mathrm{DMD}$ modes were found at the frequencies corresponding to the LOX post eigenmodes. This was contradictory to previous results in which the dominant LOX post modes were also present in the PSD spectra of point-like $\mathrm{OH}^{*}$ measurements for stable conditions. For the second load point with increased chamber 1T-mode amplitude, the expected dominant peaks in oscillatory flame radiation intensity could be observed in both the spectra of the optical probes and the DMD results.

By using DMD it was possible to extract the flame dynamics at the LOX post eigenmode frequencies. Both $\mathrm{OH}^{*}$ and blue flame radiation yielded similar results for the flame response to the LOX post acoustics and showed a longitudinal intensity distribution with a symmetrical shape. Furthermore, the highly resolved DMD mode energy distribution allowed the flame intensity fluctuation due to the LOX post $1 \mathrm{~L}$ and $2 \mathrm{~L}$ modes and the flame response to chamber acoustics to be clearly distinguished. This supports the hypothesis of Gröning et al., that the flame intensity oscillations are not only excited by the chamber acoustics, but can independently be generated by injector acoustics.

Gröning's suggestion that injector internal hydrodynamic effects could be responsible for the excitation of the LOX post modes was investigated. Heightened flame fluctuation intensity in the data collected here appears to correlate well with Strouhal number ranges where periodic vortex shedding at the LOX post throttle orifice might be expected. Thus, this kind of vortex shedding, a phenomenon described as orifice whistling, might play a role in the excitation or amplification of the LOX post modes.

\section{Acknowledgments}

Financial support has been provided by the German Research Foundation (Deutsche Forschungsgemeinschaft - DFG) in the framework of the Sonderforschungsbereich Transregio 40. The authors would like to thank the crew of the P8 test bench as well as Robert Stützer for setting up the optical diagnostics on which the results presented here are based. Thanks also to Alex Grebe and Yannik Miene for their assistance in performing the test runs.

\section{References}

[1] Harrje, D. And Reardon, F. (Eds.) (1972). Liquid Propellant Rocket Combustion Instability. National Aeronautics and Space Administration.

[2] Sutton, G.P. ANd Biblarz, O. (2010). Rocket Propulsion Elements. 8 edn. John Wiley \& Sons, New York.

[3] RAYLEIGH, J.W.S. (1878). The explanation of certain acoustical phenomena. Nature, 18(455), 319-321.

[4] YANG, V. AND ANDERSON, W. (Eds.) (1995). Liquid Rocket Engine Combustion Instability. American Institute of Aeronautics and Astronautics.

[5] Wanhainen, J.P., PARISh, H.C. AND ConRAD, E.W. (1966). Effect of propellant injection velocity on screech in 20000-pound hydrogen-oxygen rocket engine. NASA TN D-3373, National Aeronautics and Space Administration, Washington D.C.

[6] Nunome, Y., Onodera, T., Sasaki, M., Tomita, T., Kobayashi, K. And DalMON, Y. (2011). Combustion instability phenomena observed during cryogenic 
hydrogen injection temperature ramping tests for single coaxial injector elements. In: 47th AIAA/ASME/SAE/ASEE Joint Propulsion Conference \& Exhibit 2011. San Diego, California.

[7] Watanabe, D., Tamura, T., Onga, T., Manako, H., Negoro, N., Kurosu, A., KOBAYASHI, T. AND OKITA, K. (2015). Hot-fire testing of LE-X thrust chamber assembly. In: 30th International Symposium on Space Technology and Science (ISTS) 2015. Kobe, Japan.

[8] Gröning, S., HaRdi, J.S., Suslov, D. AND Oschwald, M. (2016). Injectordriven combustion instabilities in a Hydrogen/Oxygen rocket combustor. Journal of Propulsion and Power, 32, 560-573.

[9] Armbruster, W., Hardi, J.S., Suslov, D. AND Oschwald, M. (2017). Experimental investigation of self-excited combustion instabilities with injection coupling in a cryogenic rocket combustor. In: 68th International Astronautical Congress (IAC). Adelaide, Australia.

[10] GRÖNING, S. (2017). Untersuchung selbsterregter Verbrennungsinstabilitäten in einer Raketenbrennkammer. Ph.D. thesis, RWTH Aachen.

[11] Nunome, Y., Takahashi, M., Kumakawa, A., Miyazaki, K., Yoshida, S. AND ONGA, T. (2008). High-frequency flame oscillation observed at a coaxial lox/lh2 injetor element. In: 44th AIAA/ASME/SAE/ASEE Joint Propulsion Conference \& Exhibit 208. Hartforf, CT.

[12] KaWAshima, H., KobaYAShI, K. AND TOMITA, T. (2010). A combustion instability phenomenon on a LOX/Methane subscale combustor. In: 46th AIAA/ASME/SAE/ASEE Joint Propulsion Conference \& Exhibit 2010. Nashville, TN.

[13] Jensen, R.J., Dodson, H.C. AND CLAfLIN, S.E. (1989). LOX/Hydrocarbon Combustion Intability Investigation. NASA Lewis Research Center.

[14] HARDI, J.S., BeinKE, S.K., Oschwald, M. AND Dally, B.B. (2014). Coupling of cryogenic oxygen-hydrogen flames to longitudinal and transverse acoustic instabilities. Journal of Propulsion and Power, 30.

[15] Beinke, S., Tonti, F., Karl, S., Hardi, J., Oschwald, M. and Dally, B. (2017). Modelling flame response of a co-axial LOx/GH2 injection element to high frequency acoustic forcing. In: 7th European Conference for Aeronautics and Aerospace Sciences (EUCASS) 2017. Milano, Italy.

[16] Gröning, S., Suslov, D., Oschwald, M. and Sattelmayer, T. (2013). Stability behaviour of a cylindrical rocket engine combustion chamber operated with liquid hydrogen and liquid oxygen. In: 5th European Conference for Aeronautics and Space Sciences (EUCASS) 2013. Munich, Germany.

[17] Schulze, M. AND SATtelmayeR, T. (2015). Flamedynamics in supercritical $\mathrm{H} 2 / \mathrm{O} 2$ rocket combustion systems. In: SFB TRR40 Annual Report 2015. Munich, Germany.

[18] Urbano, A., Selle, L., Staffelbach, G., Cuenot, B., Schmitt, T., DUCRUIX, S. AND CANDEL, S. (2016). Exploration of combustion instability triggering using large eddy simulation of a multiple injector liquid rocket engine. Combustion and Flame, 169, 129-140.

[19] Hardi, J.S., Hallum, W.Z., Huang, C. ANd Anderson, W.E. (2016). Approaches for comparing numerical simulation of combustion instability and flame imaging. Journal of Propulsion and Power, 32, 279-294.

[20] Webster, S., HaRDI, J. AND Oschwald, M. (2016). Comparison of oxygenhydrogen combustion visualision techniques under representative conditions. In: 
Space Propulsion 2016. Roma, Italy.

[21] Rey, C., Ducruix, S., Richecoeur, F., Scouflaire, P., Vingert, L. and CANDEL, S. (2004). High frequency combustion instabilities associated with collective interactions in liquid propulsion. In: 40th AIAA/ASME/SAE/ASEE Joint Propulsion Conference and Exhibit. Fourt Lauderdale, Florida.

[22] OschwalD, M. AND KNAPP, B. (2009). Investigation of combustion chamber acoustics and its interaction with lox/h2 spray flames. Progress in Propulsion Physics, 1, 205-224.

[23] Fiala, T. and Sattelmayer, T. (2016). Assessment of existing and new modeling strategies for the simulation of oh* radiation in high-temperature flames. CEAS Space Journal, 8, 47-58.

[24] Fiala, T., Sattelmayer, T., Gröning, S., Hardi, J., Stützer, R., Webster, S. AND OSCHWALD, M. (2017). Comparison between excited hydroxyl radical and blue radiation from hydrogen rocket combustion. Journal of Propulsion and Power, 33, 490-500.

[25] FiaLA, T. (2015). Radiation from High Pressure Hydrogen-Oxygen Flames and its Use in Assessing Rocket Combustion Instability. Ph.D. thesis, Technical University of Munich, Munich, Germany.

[26] Stützer, R., Fiala, T. and Oschwald, M. (2017). The pressure dependence of optical flame emission from space propulsion-relevant hydrogen combustion. In: 7th European Conference for Aeronautics and Aerospace Sciences (EUCASS) 2017. Milano, Italy.

[27] Schmid, P.J. (2010). Dynamic mode decomposition of numerical and experimental data. J. Fluid Mech., 656, 5-28.

[28] Wierman, M., Feldman, T. And Anderson, W. (2013). Development of combustion response functions in a subscale high pressure longitudinal combustor. In: 49th AIAA/ASME/SAE/ASEE Joint Propulsion Conference. San Jose, California.

[29] Huang, C., Anderson, W.E., Harvazinski, M.E. and Sankaran, V. (2016). Analysis of self-excited combustion instabilities using decomposition techniques. AIAA Journal, 54, 2791-2807.

[30] Testud, P., Auregan, Y., Moussou, P. and Hirschberg, A. (2009). The whistling potentiality of an orifice in an confined flow using an energetic criterion. Journal of Sound and Vibration, 325, 769-780.

[31] HitT, M.A., LINEBERY, D.M., AHUJA, V. AND FREdeRICK, R.A. (2012). Experimental investigation of cavitation induced feedline instability from an orifice. In: 489th AIAA/ASME/SAE/ASEE Joint Propulsion Conference \& Exhibit. Atlanta, Georgia.

[32] Lacombe, R., Moussou, P. And Auregan, Y. (2011). Whistling of an orifice in a reverberating duct at low mach number. Journal of the Acoustical Society of America, 130, 2662-2672. 